\title{
Improving approximate extraction of functional similar regions from large-scale spatial networks based on greedy selection of representative nodes of different areas
}

\author{
${\text { Takayasu Fushimi }{ }^{*} \text { (D), Kazumi Saito }}^{2,3,4}$, Tetsuo Ikeda ${ }^{4}$ and Kazuhiro Kazama ${ }^{5}$
}

\author{
${ }^{*}$ Correspondence: \\ takayasu.fushimi@gmail.com \\ ${ }^{1}$ Tokyo University of Technology, \\ 1404-1 Katakuramachi, Hachioji city, \\ Tokyo 192-0982, Japan \\ Full list of author information is \\ available at the end of the article
}

\begin{abstract}
Dividing a geographical region into some subregions with common characteristics is an important research topic, and has been studied in many research fields such as urban planning and transportation planning. In this paper, by network analysis approach, we attempt to extract functionally similar regions, each of which consists of functionally similar nodes of a road network.

For this purpose, we previously proposed the Functional Cluster Extraction method, which takes a large amount of computation time to output clustering results because it treats too many high-dimensional vectors. To overcome this difficulty, we also previously proposed a transfer learning-based clustering method that selects approximate medoids from the target network using the $K$ medoids of a previously clustered network and divides all the nodes into $K$ clusters. If we select an appropriate network with similar structural characteristics, this method produces highly accurate clustering results. However it is difficult to preliminarily know which network is appropriate. In this paper, we extend this method to ensure accuracy using the $K$ medoids of multiple networks rather than a specific network. Using actual urban streets, we evaluate our proposed method from the viewpoint of the improvement degree of clustering accuracy and computation time.
\end{abstract}

Keywords: Spatial network, Functional similarity, Node clustering, Greedy algorithm, Transfer learning

\section{Introduction}

From a geographical area, extracting territorial unit each of which has the homogeneity in terms of physical aspects, cultural aspects and so forth, is an important research task in geography like urban planning and transportation planning. There exist some studies attempting to divide a given area into subregions or to find similar regions having common structural patterns by multivariate analysis approaches (Berry 1964; Grigg 1965; Berry 1968) and by network analysis approaches (Zhang et al. 2011; Farmer and Fotheringham 2011; Yin et al. 2017; Chen et al. 2018). Without being limited to hyperlink networks and user-related networks in SNS, various types of networks like road networks and electric power networks have been analyzed (Burckhart and Martin 2012; Crucitti et al. 2006; Montis et al. 2007; Opsahl et al. 2010; Park and Yilmaz 2010; Wang et al. 2012).

(c) The Author(s). 2018 Open Access This article is distributed under the terms of the Creative Commons Attribution 4.0 International License (http://creativecommons.org/licenses/by/4.0/), which permits unrestricted use, distribution, and reproduction in any medium, provided you give appropriate credit to the original author(s) and the source, provide a link to the Creative Commons license, and indicate if changes were made. 
In a network, each node plays some sort of function for it. Examples of functions include manager and general employee in a company network and urban and suburban areas in a road network. Discovering node roles or extracting functionally similar nodes from social networks has become an important task in network analysis (Henderson et al. 2011; Henderson et al. 2012; Rossi et al. 2012; 2013; Gilpin et al. 2013; Rossi and Ahmed 2015).

For the similar purpose, we previously proposed the Functional Community/Cluster Extraction (FCE) method that extracts functionally similar nodes, which we call functional cluster, from social or web networks (Fushimi et al. 2012) and from spatial networks like urban streets (Fushimi et al. 2016a). The FCE method first calculates a feature vector for each node that is generally represented as a high-dimensional vector. Then it divides all the vectors into $K$ clusters, each of which consists of functionally similar nodes, using the $K$-medoids method based on a greedy algorithm.

Figures $1 \mathrm{a}$ and $\mathrm{b}$ respectively indicate the main landmarks in and around the area of the Shizuoka network and the functional clusters extracted by the FCE method, where the node colors stand for the functional clusters at $K=5$. From these figures, the blue regions roughly correspond to the city centers of this network, the red regions approximately correspond to suburban areas, the green regions contain many nodes in the agricultural areas or mountain foothills, the yellow regions contain many nodes with long, winding roads, which lead to other towns over the mountainous areas, and the pink regions roughly correspond to small villages with sparse population.

However, the FCE method takes a large amount of computation time to output clustering results because it treats so many high-dimensional vectors for a large-scale network. To overcome this difficulty, we proposed an accelerated version of a greedy algorithm for $K$-medoids clustering, which produces identical results to the original FCE method, by equipping it with some pruning techniques (Fushimi et al. 2016b). For further acceleration, by focusing on the structural similarity of urban streets and regarding them as spatial networks, we proposed a transfer learning-based method (Fushimi et al. 2017c), which approximates medoid vectors using an already clustered network. We call this a source domain network (source network). By using approximates medoid vectors, the method divides all the nodes of a target network into $K$ clusters. If we select an appropriate network with similar functional and structural characteristics, this method produces highly accurate clustering results. To obtain high approximation accuracy, we must know in advance which network is suitable as a source network.

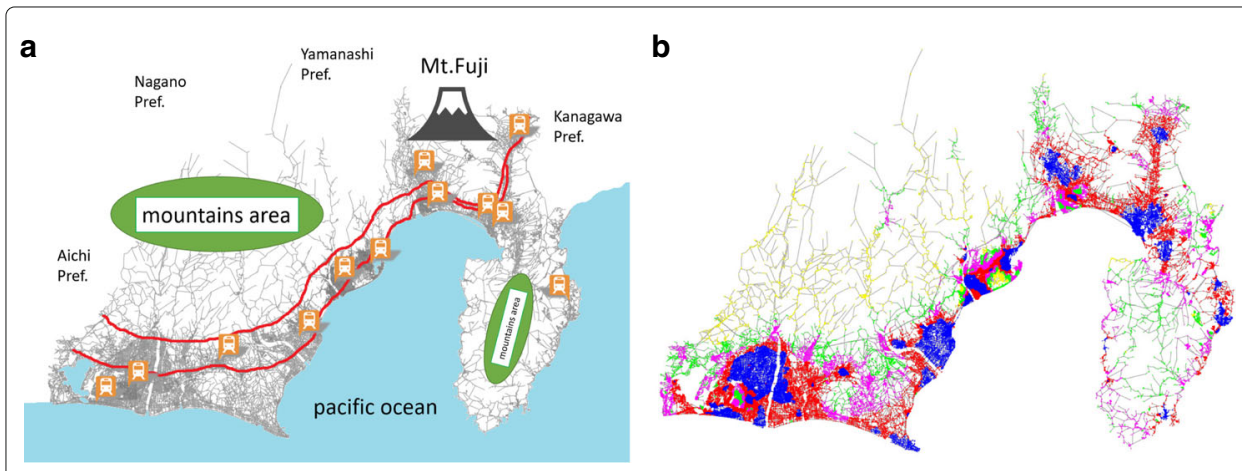

Fig. 1 Visualization results of Shizuoka $\mathbf{a}$ Landmarks $\mathbf{b}$ Results of FCE method $(K=5)$ 
Furthermore, a target spatial network naturally consists of some functional regions, each of which resembles those of other networks rather than a specific network. From our previous experiments (Fushimi et al. 2016a), we learned that for all of the six cities used in our experiments, the 1st, 2nd, and 3rd functional clusters share commonly similar characteristics. On the other hand, the 4th and 5th functional clusters do not, because they reflect geographical restrictions and/or the historical and the cultural backgrounds of individual cities. For example, in all the networks, the 3rd functional regions significantly contain many nodes whose degree is four, which implies that they are downtown regions with a lattice structure like Manhattan.

Therefore, in this paper, based on these observations, we extend the above transfer learning-based method (Fushimi et al. 2017c) by using the $K$ medoids of more than one source network and use $K$ medoids of $M$ source networks, where the total number of medoids is $M K$. For a target network that consists of $N$ nodes, each of whose function is represented as a high-dimensional vector, we select $M K$ candidates of approximate medoids from $N$ nodes based on the cosine similarities among the vectors of the $M K$ medoids and the $N$ nodes. Then we extract the $K$-approximate medoids from the $M K$ candidates based on a greedy algorithm, which selects the nodes with the largest marginal gain of the objective function. In addition, to improve the objective function value, we introduce an update mechanism like $k$-means algorithm to the approximate medoids, which we call Divided Improvement (DI).

The rest of this paper is organized as follows. After explaining related work in "Related work" section, we revisit the extraction method of functional clusters in "FCE method revisit" section. Then in "Simple selection of approximate medoids from a single source network" section, we describe the transfer learning-based method and our proposed method in "Greedy selection of approximate medoids from multiple source networks" section. After explaining the network dataset in "Spatial network dataset" section, in "Evaluation of computation time" section, we evaluate its computational performance. In "Evaluation of clustering accuracy" section, we evaluate the accuracy of our proposed algorithm and discuss the objective function value of our proposed method and other comparison methods in "Evaluation of objective function value" section. Finally, we conclude in "Conclusion" section.

\section{Related work}

Extracting node functions from a network is one important research topic, especially in sociology. Concept and extraction algorithms of regular equivalence (Everett and Borgatti 1994) and structural equivalence (Lorrain and White 1971) have been proposed. These concepts focus on such local structures as relationships with neighboring nodes. However, the functional vector of the FCE method reflects not only the local structure but also the global structure by changing the number of dimensionalities $S$ of functional vectors. More recently, many role discovery techniques have been proposed (Henderson et al. 2011; 2012; Rossi et al. 2012; 2013; Gilpin et al. 2013; Rossi and Ahmed 2015). These techniques are intended for scale-free networks whose degree distribution follows a power law like a social network. In this paper, we focus on spatial networks like urban streets, and since the upper limit of the degree is relatively small, these techniques cannot be straightforwardly applied to spatial networks. 
Studies on dividing a given geographical area into some functional regions or uniform regions have been conducted in geography. Though notion of these regions is somewhat different from our functional clusters, they are similar in terms of extracting certain regions with common characteristics. To extract these regions, multivariate and network analysis methods were proposed (Berry 1964; Grigg 1965; Berry 1968; Zhang et al. 2011; Farmer and Fotheringham 2011; Yin et al. 2017; Chen et al. 2018). As a network analysis based approach, Zhang et al. analyzed topological structure of road networks and distinguished these structures into some patterns (Zhang et al. 2011). Though the study was based on the existing TAZ (Traffic Analysis Zone) delineation, Zhang et al. mentioned that how to decide the analysis unit is an important task and it should be studied as a future work. Our FCE method extracts functionally similar regions each of which could be regarded as an unit with the similar road pattern including lattice in a city center, loops and lollipops in a residential district, and winding road in a mountainous area (Fushimi et al. 2016a). Farmer and Fotheringham applied the community detection method, which is proposed by Newman (2004), to networks of travel-to-work flows, and found internally well connected and relatively cohesive regions (Farmer and Fotheringham 2011). Note that their method does not consider whether distant or disconnected regions have the similar function or not, unlike our FCE method. In order to delineate urban boundaries based on human movements, Yin et al. adopted the community detection method, Infomap (Rosvall and Bergstrom 2007), to a directed weighted network, where nodes and weighted links respectively represent underlying urban regions and Twitter users' displacements on them (Yin et al. 2017). While the method utilizes actual human movements obtained from geo-located tweets, our FCE method focuses on artificial ones based on a random walk model on a road network considering a situation that these movements data cannot be obtained.

Research on community detection or graph clustering is another major stream of complex network analysis. As mentioned above, we adopt a method (Fushimi et al. 2012) to extract functional clusters. This is because representative methods for extracting communities as densely connected subnetworks, which include the Newman clustering method based on a modularity measure (Newman 2004), cannot directly deal with such functional properties. The conventional concept of a subnetwork that is connected densely, for example, $k$-core (Seidman 1983) and $k$-clique (Palla et al. 2005), cannot also be used for this purpose. Namely, we naturally anticipate that these representative methods have an intrinsic limitation for extracting functional similar nodes. Directly applying these conventional methods to a spatial network is difficult, since the maximum degree of nodes in each network is generally limited to a relatively small number, because it is unlikely that densely connected subnetworks will appear in these networks.

The process of Power Iteration Clustering (PIC) (Lin and Cohen 2010), which is one scalable graph clustering method, and those of our FCE method create a similar framework. The PIC method utilizes vectors obtained by truncated power iteration on a matrix, which resembles the normalized random-walk Laplacian matrix, by changing initial vector and then dividing all the nodes into $K$ clusters with the $K$-means algorithm. On the other hand, our FCE method utilizes power iteration on a random-walk transition matrix for generating a feature vector of each node $u$, whose elements are the midstream probabilities of random-walk until the values converge. Therefore, for a large-scale spatial network, the dimensionality of $S$ tends to be large. 
In this paper, we focus on the FCE method using the $K$-medoids clustering method that divides all the nodes into functionally similar nodes by the greedy maximization of the objective function. Typical sampling algorithms like Jiang et al. (2002) and Aggarwal et al. (2009) can cluster large datasets. Another previous work (Jiang et al. 2002) focused on the fractal structure of the dataset and extracted a subset of significant size that holds the entire dataset structure. However, because the approximate centers or clusters are computed from stochastically chosen, relatively small objects, the accuracy of the results is not guaranteed. Since our proposed method focuses on the similar structure of road networks, we expect to obtain relatively higher accuracy than the above sampling methods.

\section{FCE method revisit}

We proposed the Functional Cluster Extraction (FCE) method that just extracts functionally similar node groups from the topological structure of a given network (Fushimi et al. 2012). The FCE method consists of two steps: the calculation of the feature vector for each node and the clustering of these vectors. For undirected network $G=(V, E)$, where $V$ and $E$ respectively stand for sets of nodes and undirected links, the FCE method considers the random-walk process, where the initial probability of each node is set to a uniform value.

Concretely, for each node $u \in V$, we define the probability at iteration step $s$ of randomwalk process

$$
y_{s}(u)=\sum_{v \in \Gamma(u)} \frac{y_{s-1}(v)}{|\Gamma(v)|},
$$

where $\Gamma(u)=\{v \mid(u, v) \in E\}$ is the adjacent node set of node $u$. Random-walk probability $y_{s}(u)$ has properties where $y_{s}(u) \geq 0$ and $\sum_{u \in V} y_{s}(u)=1$, and the initial probability of each node $u$ is set to $y_{0}(u)=1 / N$, where $N$ is the number of nodes and $N=|V|$. Then we define the probability vector $\mathbf{y}_{s}$ whose element is the probability of each node at iteration step $s$. This model can be regarded as a special version of PageRank where the teleportation jump probability is set to 0 .

Now we define the $S$-dimensional vector of node $u$ :

$$
\mathbf{x}_{u}=\left(y_{1}(u), \cdots, y_{S}(u)\right),
$$

where $S$ denotes the final step of the random-walk iterations. Hereafter, $\mathbf{x}_{u}$ is called the functional vector of node $u$.

Next, for each pair of nodes, we define the functional similarity calculated by the cosine similarity,

$$
\rho(u, v)=\left\langle\frac{\mathbf{x}_{u}}{\left\|\mathbf{x}_{u}\right\|}, \frac{\mathbf{x}_{v}}{\left\|\mathbf{x}_{v}\right\|}\right\rangle,
$$

between the functional vectors of the corresponding nodes. Then the FCE method divides all the nodes into $K$ groups of functional clusters by employing the $K$-medoids algorithm (Vinod 1969) due to its robustness. Formally, we maximize the following objective function with respect to set of medoids $R \subset V$ :

$$
f(R)=\sum_{v \in V} \max _{r \in R} \rho(v, r) .
$$


To maximize this objective function, we employ a greedy algorithm based on the following marginal gain of each node:

$$
g(w ; R)=f(R \cup\{w\})-f(R)=\sum_{v \in V \backslash R} \max \{\rho(v, w)-\mu(v ; R), 0\},
$$

where $w$ is a candidate node of the medoid, $R$ is the set of already selected medoids, and $\mu(v ; R)=\max _{r \in R} \rho(v, r)$ is the similarity between node $v$ and the most similar medoid, $r$. The greedy algorithm selects a node that gives the highest marginal gain with respect to the already selected medoid set as the next medoid (representative vector):

$$
r_{k}=\underset{w \in V \backslash R}{\arg \max } g(w ; R) .
$$

Therefore, we obtain a node with the most central functional vector as the first medoid and a node with the most different functional vector from the first medoid as the second medoid. That is, in the FCE method, each medoid is a representative node of each functionally different area.

For a large-scale network, however, the $K$-medoids method based on a greedy algorithm needs a huge amount of computation time, $O\left(N^{2} S\right)$, to calculate the functional similarity of all the node pairs, and it is also difficult to store all $N(N-1) / 2$ similarities on the main memory. Thus we have to re-calculate all the similarities in all the $K$-greedy steps of $K$-medoids clustering, and the calculation cost becomes $O\left(K N^{2} S\right)$.

\section{Simple selection of approximate medoids from a single source network}

Spatial networks like urban streets have similar topological structures among distant areas (Wang et al. 2012; Jiang et al. 2014; Fushimi et al. 2016a). By exploiting this knowledge, we propose a fast method of the clustering phase in the FCE method based on transfer learning, which utilizes a set of $K$ medoids in a source domain network (source network) for clustering all the nodes of a target domain network (target network). In our context, source and target networks respectively stand for a network whose nodes have been already divided into $K$ clusters and a network whose nodes have not been clustered. Hereafter, this method is called the Transfer Learning (TL) method.

Formally, let $V$ and $\mathbf{X}$ be a set of nodes and a set of functional vectors of a target network, and let $\mathbf{Z}=\left\{\mathbf{z}_{1}, \ldots \mathbf{z}_{K}\right\}$ be a set of medoid vectors in a source network, all of which were previously selected by $K$-medoids clustering with a greedy algorithm in the FCE method. Then the TL method selects $K$-approximate medoids $R^{\prime}=\left\{r_{1}^{\prime}, \ldots, r_{K}^{\prime}\right\} \subset V$ of the target network as follows:

$$
r_{k}^{\prime}=\underset{v \in V}{\arg \max }\left\langle\frac{\mathbf{z}_{k}}{\left\|\mathbf{z}_{k}\right\|}, \frac{\mathbf{x}_{v}}{\left\|\mathbf{x}_{v}\right\|}\right\rangle
$$

The TL method extracts $K$-approximate medoids from the $N$ nodes in the target network by calculating the cosine similarities between the $S$-dimensional functional vectors of all pairs of $K$ medoids in the source network and the $N$-medoid candidates in the target network. Then the TL method divides the $(N-K)$ remaining nodes in the target network into $K$-approximate functional clusters by calculating the cosine similarities between the $S$-dimensional functional vectors of all the pairs of the $K$-approximate medoids and the $(N-K)$ remaining nodes in the target domain. Therefore the total computational cost becomes $O(K N S)$. 


\section{Greedy selection of approximate medoids from multiple source networks}

When using the TL method, we obtain good approximation results by selecting an appropriate network as a source network. But it is difficult to know in advance which network is suitable. In addition, generally speaking, a network contains regions that resemble multiple networks rather than a single network. Figure 2 shows an example of the similarities of the five medoids of a target network and the five medoids of four source networks. In this example, the first medoid, $r_{1}$, of the target network is a node of a suburban area and most resembles the first medoid of source network NW3. Similarly to the above, the second medoid, $r_{2}$, of the target network is a node of an agricultural area and most closely resembles the second medoid of source network NW4. That is, the target network has similar regions with source networks NW1, NW3, and NW4 rather than a specific source network.

Based on these considerations, we propose a greedy selection method that selects $K$ approximate medoids from multiple networks as source domain rather than a single network. Hereafter, this method is referred to as the Greedy Selection (GS) method.

Formally, let $V$ and $\mathbf{X}$ be a set of nodes and a set of functional vectors of a target network, and let $\mathbf{Z}^{(m)}=\left\{\mathbf{z}_{1}^{(m)}, \ldots \mathbf{z}_{K}^{(m)}\right\}$ be a set of medoid vectors in one source network $G_{m}$, where these medoid vectors were previously selected by $K$-medoids clustering with a greedy algorithm in the FCE method. When given the medoid vectors of $M$ source networks $G_{1}, \ldots, G_{M}$, the GS method selects $K$-approximate medoids $R^{\prime}=\left\{r_{1}^{\prime}, \ldots, r_{K}^{\prime}\right\} \subset V$ of the target network in the following steps:

1. $\quad$ Select $K$ sets of medoid candidates $U(k) \subset V$;

2. Select $K$-approximate medoids $R^{\prime}$ from each set $U(k)$ of medoid candidates.

In the first step, the GS method selects $M K$ nodes as medoid candidates by calculating the cosine similarity for all the pairs of the $K$-medoid vectors in the $M$ source networks and the $N$ vectors:

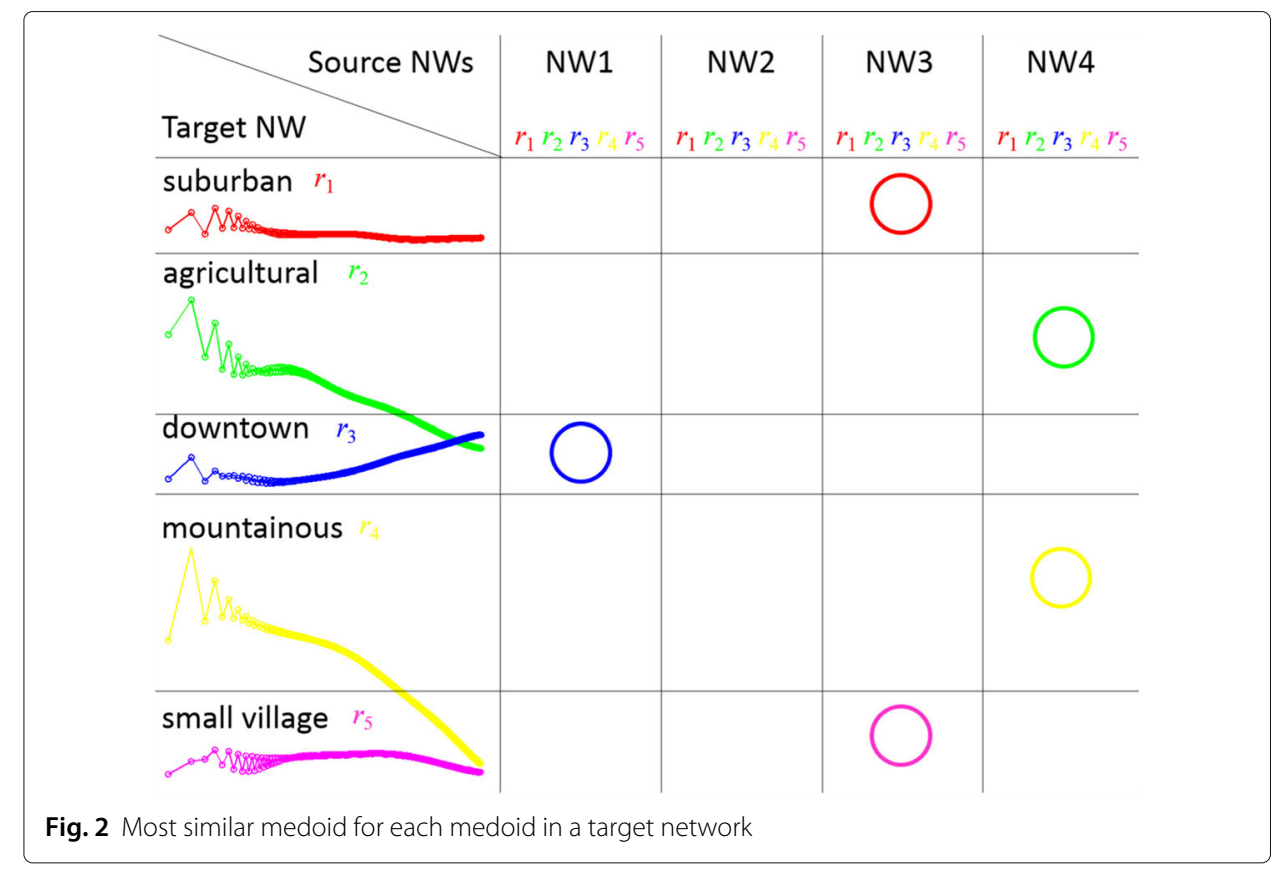




$$
u(k \mid m)=\underset{u \in V}{\arg \max }\left\langle\frac{\mathbf{z}_{k}^{(m)}}{\left\|\mathbf{z}_{k}^{(m)}\right\|}, \frac{\mathbf{x}_{u}}{\left\|\mathbf{x}_{u}\right\|}\right\rangle .
$$

Then for each $k$, we define $U(k)=\{u(k \mid 1), \ldots, u(k \mid M)\}$ as a set of the medoid candidates of $k$. In the second step, the GS method selects the $K$-approximate medoids by maximizing the objective function in Eq. (1) with respect to the set of approximate medoids $R^{\prime} \subset U$, where $U$ is a union of the set of medoid candidates, $U=\bigcup_{k=1}^{K} U(k)$. To maximize the objective function, we employ a greedy algorithm similarly to the $K$ medoids method in the original FCE method, where we select approximate medoid $r_{k}^{\prime}$ of the $k$ step as follows:

$$
r_{k}^{\prime}=\underset{u \in U(k)}{\arg \max } g\left(u ; R^{\prime}\right)=\underset{u \in U(k)}{\arg \max } \sum_{v \in V \backslash R^{\prime}} \max \left\{\rho(v, u)-\mu\left(v ; R^{\prime}\right), 0\right\},
$$

where $\rho(\cdot)$ and $\mu(\cdot)$, which are used in Eq. (4), are the same as those defined in Section 3. The difference between the greedy $K$-medoids method in the original FCE method (Eq. (3)) and the GS method (Eq. (4)) is the size of the search space of the marginal gain, and the former is the total number of nodes $N=|V|$, and the latter is the number of source networks $M=|U(k)| \ll N$.

The GS method extracts $M K$-medoid candidates from the $N$ nodes in the target network by calculating the cosine similarities between the $S$-dimensional functional vectors of all the pairs of the $M K$-medoids in the source networks and the $N$ nodes in the target network. Next it extracts the $K$-approximate medoids from the $M$ candidates at each of the $K$-greedy steps by calculating the cosine similarities between the $S$-dimensional functional vectors of all the pairs of $M$ candidates and $N$ nodes. Therefore, the total computational cost becomes $O(M K N S)$, which is slightly larger than that of the TL method $O(K N S)$ but much smaller than that of the original FCE method $\left(K N^{2} S\right)$.

\section{Spatial network dataset}

In our experiments, we used the following 15 cities extracted from Open Street Map $(\mathrm{OSM})^{1}$ and Digital Road Map (DRM) data. We extracted all the intersections and the roads of each city and constructed a spatial network with intersections as nodes and the roads between them as links. To simplify our analyses, we deleted nodes that represent the curved segments of highways by directly connecting both sides of the deleted ones.

Table 1 shows the basic statistics of the networks for the 15 cities, where $C$ and $L$ respectively denote the averages of the clustering coefficients and the shortest path length over each network. Although the numbers of nodes and links $|V|$ and $|E|$ are substantially different, the degree distributions defined by $p_{j}$ as well as $C$ and $L$ are quite similar as the common characteristics of these spatial networks.

\section{Evaluation of computation time}

We experimentally evaluated the efficiency of our proposed method, the GS method, in terms of its computation time by comparing the following three baseline methods including our previous methods: the first method, which only employs the Lazy Evaluation (LE) technique (Leskovec et al. 2007), is referred to as the (a) LE method; the second method, which employs LE, medoid pruning, and outlier pivot pruning techniques (Fushimi et al. 2016b), is called the (b) Pivot Pruning (PP) method, where we set the number of outlier 
Table 1 Basic network statistics

\begin{tabular}{llllllllll}
\hline City & $|V|$ & $|E|$ & $p_{1}$ & $p_{2}$ & $p_{3}$ & $p_{4}$ & $p_{>4}$ & $C$ & $L$ \\
\hline Aichi & 259,915 & 402,465 & .065 & .070 & .574 & .285 & .006 & 0.04 & 98.71 \\
Barcelona & 66,790 & 99,387 & .103 & .031 & .659 & .201 & .006 & 0.06 & 53.07 \\
Brasilia & 95,811 & 136,955 & .133 & .025 & .694 & .146 & .002 & 0.04 & 92.94 \\
Chiba & 227,791 & 335,564 & .116 & .003 & .703 & .177 & .002 & 0.04 & 99.73 \\
Ibaraki & 172,892 & 263,075 & .083 & .002 & .708 & .205 & .003 & 0.04 & 111.02 \\
Kanagawa & 295,151 & 402,576 & .192 & .051 & .597 & .155 & .005 & 0.04 & 129.17 \\
Kyoto & 88,800 & 128,601 & .099 & .090 & .633 & .174 & .004 & 0.07 & 103.43 \\
New York & 325,962 & 466,510 & .159 & .033 & .600 & .204 & .004 & 0.04 & 103.14 \\
Osaka & 261,939 & 375,750 & .133 & .066 & .606 & .190 & .006 & 0.05 & 91.32 \\
Saitama & 230,408 & 351,811 & .084 & .001 & .695 & .217 & .003 & 0.04 & 81.04 \\
San Francisco & 110,700 & 156,821 & .173 & .037 & .583 & .199 & .009 & 0.05 & 79.43 \\
Seoul & 103,444 & 150,822 & .111 & .078 & .605 & .198 & .008 & 0.04 & 43.99 \\
Shizuoka & 110,925 & 162,322 & .121 & .070 & .576 & .228 & .005 & 0.05 & 83.09 \\
Tokyo & 340,919 & 485,858 & .163 & .034 & .599 & .197 & .006 & 0.03 & 126.19 \\
Washington D.C. & 24,564 & 38,053 & .096 & .028 & .571 & .293 & .012 & 0.05 & 51.89 \\
\hline
\end{tabular}

pivots to 10; and the third method, based on the Transfer Learning (TL) technique mentioned in Section 4, is called the (c) TL method (Fushimi et al. 2017c). In our experiments, we changed the number of medoids, $K$, from 2 to 10, the number of dimensionalities of the functional vectors, $S$, to $10,100,1000$, and 10,000 , and set the number of source networks, $M=14$, in the GS method. We performed our experiments on a computer system equipped with an Xeon processor E5-2697 2.7 GHz and 256-GB main memory.

Figure 3 shows the computation times of the above methods, where TL is the average computation times that used each of the 14 networks as a source network. We show the results of eight randomly selected networks from Table 1 by setting the number of dimensionalities of each functional vector as $S=10,000$, where the horizontal and vertical axes respectively stand for the number of medoids (clusters) and the computation times with a logarithmic scale. Note that we only show the computation time of the clustering phase without including the calculations of the functional vectors. From Fig. 3, for all the networks, the methods with the approximate medoids, TL and GS, worked extraordinarily

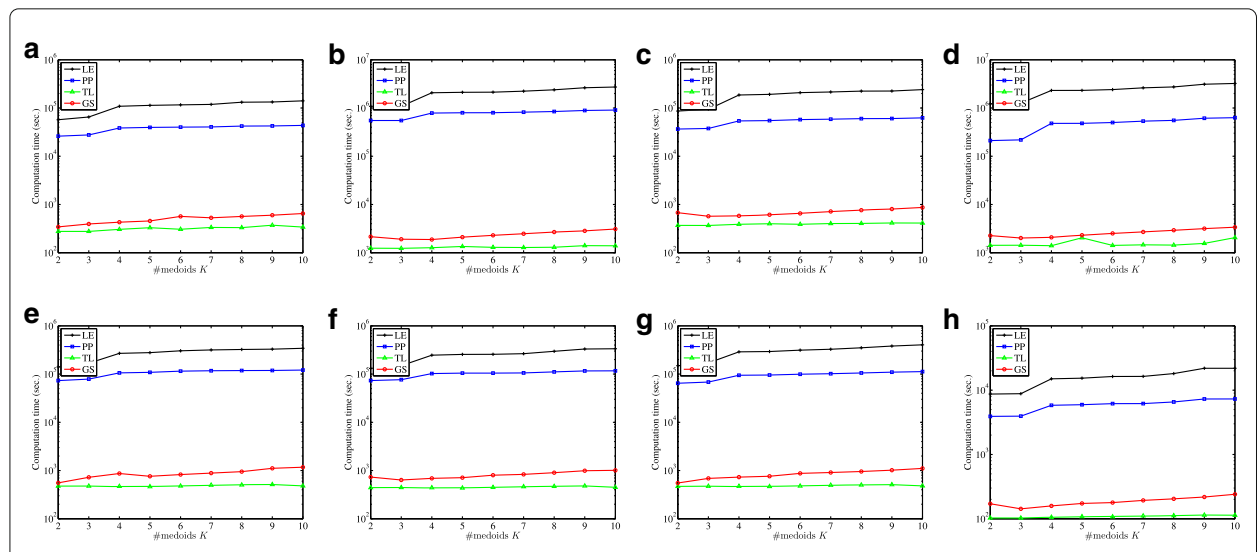

Fig. 3 Computation time w.r.t. number of medoids $K(S=10,000)$ a Barcelona $\mathbf{b}$ Kanagawa c Kyoto $\mathbf{d}$ New York e San Francisco f Seoul g Shizuoka h Washington D. C 
faster than the LE and PP methods. Furthermore, the computation times of the former two methods barely changed as the number of medoids $K$ becomes larger.

Figure 4 shows the computation times of the four methods for the eight networks (as in Fig. 3) by setting the number of medoids to $K=10$, where the horizontal axis stands for the number of dimensionalities of the functional vectors. From Fig. 4, for all the networks, the TL and GS methods returned clustering results much faster than the LE and PP methods, regardless of the number of dimensionalities of vectors.

\section{Evaluation of clustering accuracy}

Since the GS and TL methods select approximate medoids based on medoids of other networks, the clustering results somewhat differ from the original FCE method, unlike the LE and PP methods. Thus, in this section, we evaluated the GS method's performance in terms of clustering accuracy and compared it to the TL method's average accuracy. In the FCE method, since the meaning of the extracted order of the functional medoids (clusters) is important, we employ an accuracy measure widely used in multiclass classification, $A C C=\frac{1}{M} \sum_{k=1}^{K} M_{k k}$, calculated by the sum of the diagonal elements of confusion matrix $M_{k k}$ and the total number of classifications, $M=\sum_{k=1}^{K} \sum_{h=1}^{K} M_{k h}$. In our experiments, we regard the class obtained by the FCE method as the actual one and the one obtained by the TL and GS methods as the estimated one.

First, we show the accuracy of the TL methods for each pair of target and source networks in Fig. 5, where the horizontal and vertical axes stand for the target and source networks, respectively.

From Fig. 5, for each target network, the accuracy shows various values from low to high. For example, for the Aichi network at $K=5$, the highest accuracy $(A C C=0.92)$ can be obtained using Chiba as a source network, and on the other hand, the lowest accuracy $(A C C=0.23)$ can be obtained using Tokyo. These results indicate that high accuracy can only be obtained when selecting the appropriate network as the source network.

In addition, even though we select the Washington D.C. network as a source network, which is the smallest one used in our experiments, high accuracy can be obtained for the New York, the Seoul and the Shizuoka networks. These observations suggested that clustering accuracy does not depend on the scales of source and target networks. However

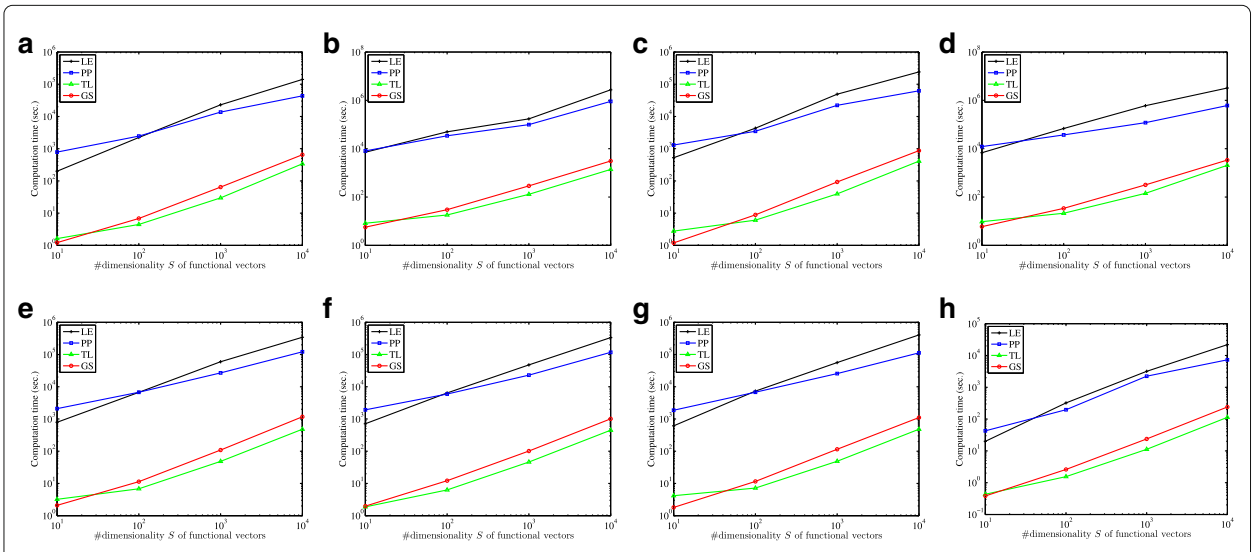

Fig. 4 Computation time w.r.t. number of dimensionalities $S(K=10)$ a Barcelona $\mathbf{b}$ Kanagawa c Kyoto d New York e San Francisco f Seoul g Shizuoka h Washington D. C 

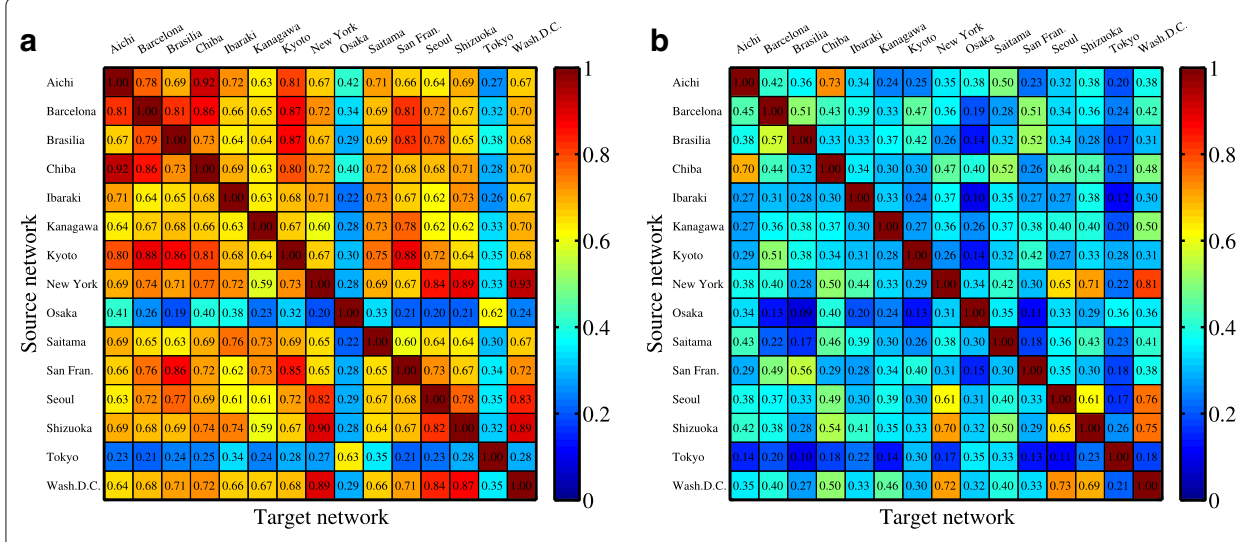

Fig. 5 Clustering accuracy $(S=10,000)$ a $K=5 \mathbf{b} K=10$

we conjecture that the TL method does not work well in the case that a source network is too small to contain only uniform functional region such as a lattice.

In Fig. 6, we plotted the GS method's accuracy as a red line and the the average accuracy and the range (maximum-minimum) of the TL method as a green error bar, with respect to the number of medoids. From Fig. 6, for all the networks, the accuracy of the GS methods is significantly higher than the average accuracy of the TL methods and somewhat higher than their maximum accuracy at almost all the points. These results confirmed that by combining the medoids of multiple source networks, clustering results with better accuracy can be obtained rather than using a specific source network.

\section{Evaluation of objective function value}

In this section, we evaluated the GS method in terms of objective function values. When we select more adequate nodes as medoids, the value of the objective function (Eq. 1) becomes larger. Therefore, we compare the objective function values of the TL and GS methods. Furthermore, to improve the quality of the clustering results, we introduce an update mechanism that is like $k$-means to the TL and GS methods, which we call Divided Improvement (DI).

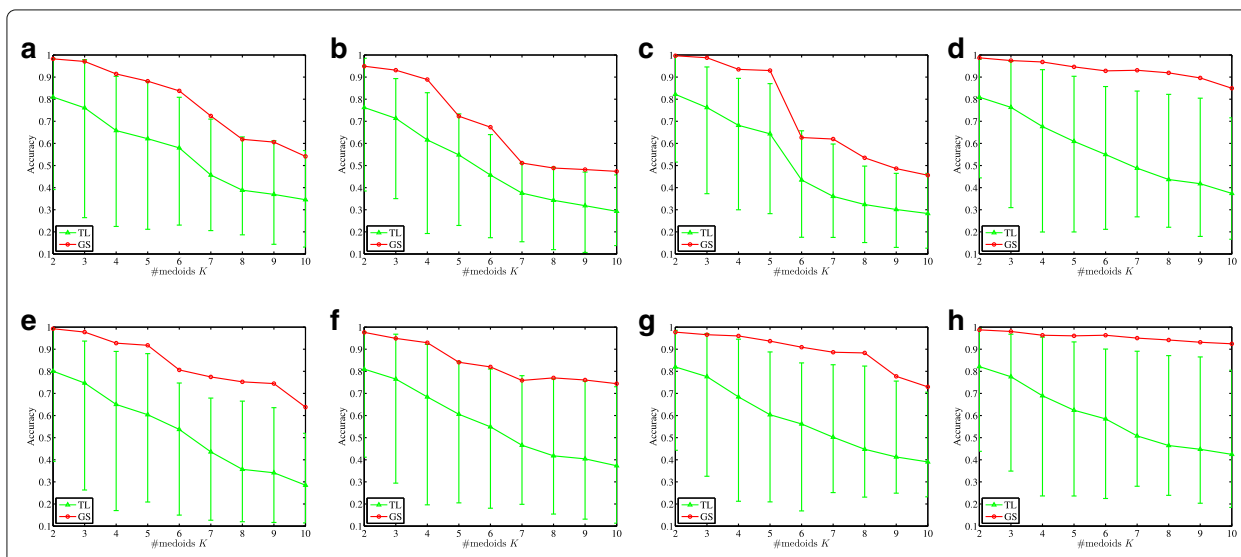

Fig. 6 Clustering accuracy w.r.t. number of medoids $K(S=10,000)$ a Barcelona b Kanagawa c Kyoto d New York e San Francisco f Seoul $\mathbf{g}$ Shizuoka h Washington D. C 
Formally, let $\mathbf{X}, R^{\prime}=\left\{r_{1}^{\prime}, \ldots, r_{K}^{\prime}\right\}$, and $V(k)=\{u \mid c(u)=k\} \subset V$ be the set of functional vectors, the set of approximate medoids obtained by the TL or GS methods, and the set of nodes that belongs to the $r_{k}^{\prime}$ 's cluster, respectively, where $1 \leq c(u) \geq K$ stands for the cluster label of node $u$. First, for the $k$-th cluster $V(k)$, we select the most central node $\hat{r}_{k}$ whose sum of cosine similarity with the other nodes in the cluster is the highest:

$$
\hat{r}_{k}=\underset{u \in V(k)}{\arg \max } \sum_{v \in V(k)} \rho(v, u)=\underset{u \in V(k)}{\arg \max } \sum_{v \in V(k)}\left\langle\tilde{\mathbf{x}}_{v}, \tilde{\mathbf{x}}_{u}\right\rangle=\underset{u \in V(k)}{\arg \max }\left\langle\sum_{v \in V(k)} \tilde{\mathbf{x}}_{v}, \tilde{\mathbf{x}}_{u}\right\rangle,
$$

where each functional vector is normalized as $\tilde{\mathbf{x}}_{u} \leftarrow \mathbf{x}_{u} /\left\|\mathbf{x}_{u}\right\|$. If selected central node $\hat{r}_{k}$ is different from approximate medoid $r_{k}^{\prime}$, we update the medoid as $r_{k}^{\prime} \leftarrow \hat{r}_{k}$. This medoid update takes a computational cost of $O(N S)$. Second, we divide the $(N-K)$ remaining nodes into $K$ clusters like Voronoi Tessellation with a computational cost of $O(K N S)$. By repeating these steps until not all of the medoids change, we improve the clustering quality. Hereafter, the TL and GS methods equipped with the DI technique are called $\mathrm{TL}+\mathrm{DI}$ and GS+DI, respectively.

Figure 7 shows the ratio of the objective function values of four methods, TL, GS, $\mathrm{TL}+\mathrm{DI}$, and GS+DI, and that of the original FCE method with respect to the number of medoids $K$, where the vertical axis is a logarithmic scale. From Fig. 7, the objective function values are improved by introducing the DI technique at almost all the number of medoids.

\section{Conclusion}

In this paper, we quickly and accurately extracted functionally similar regions from largescale spatial networks, based on a transfer learning technique that utilizes previously selected representative nodes (medoids) in a different area. With the transfer learning method developed in our previous study, we proposed the GS method by extending the source domain from one specific network to multiple networks and greedily selecting appropriate medoids from these source networks based on the marginal gain of the objective function. One of the virtues of the GS method is that when using it, we need not to know in advance which network is adequate as a source domain. From experimental evaluations with real spatial networks, we confirmed that our proposed method, GS, outputs
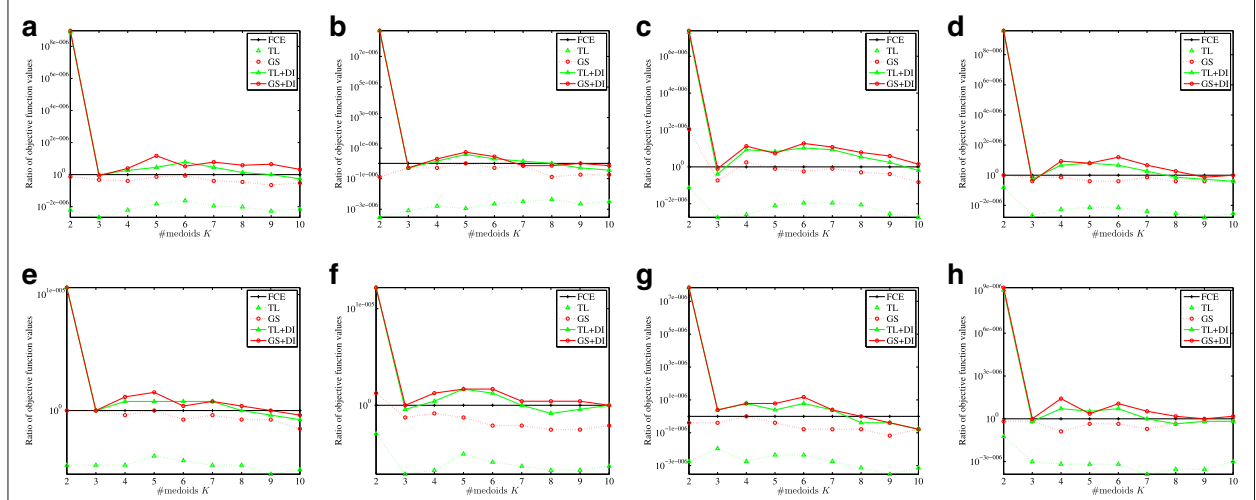

Fig. 7 Ratio of objective function values w.r.t. number of medoids $K(S=10,000)$ a Barcelona $\mathbf{b}$ Kanagawa $\mathbf{c}$ Kyoto d New York e San Francisco f Seoul g Shizuoka h Washington D. C 
more accurate clustering results than our existing one, TL, while still maintaining almost the same calculation speed.

Our proposed method is potentially applicable to some kind of networks other than spatial networks. For instance, functions like president, manager, chief and general employee commonly exist in employee networks of some companies. To extract these functions from a target employee network, our method utilizes representative functions of other companies. However, in order to confirm this claim, we need further experiments in our future study.

Therefore, in the future, we will conduct further experiments using social or web networks in addition to spatial networks.

\title{
Endnote
}

${ }^{1}$ https://mapzen.com/data/metro-extracts

Acknowledgments

We thank Prof. Seiya Okubo of the University of Shizuoka, Shizuoka, Japan, for supporting computation environments.

Funding

All authors are grateful for the financial support from JSPS Grant-in-Aid for Scientific Research (No.17H01826).

Availability of data and materials

The raw datasets used and analysed during the current study are available from an Open Street Map (OSM) site, https:// mapzen.com/data/metro-extracts, and Digital Road Map (DRM) data, http://www.drm.jp/english/drm/e_index.htm.

\section{Authors' contributions}

TF performed the research and wrote the article. KS contributed to designing the proposed method. TI contributed preparation of experimental data and part of experimental evaluations. KK contributed survey of related work and part of experimental evaluations. All authors read and approved the final manuscript.

\section{Competing interests}

The authors declare that they have no competing interests.

\section{Publisher's Note}

Springer Nature remains neutral with regard to jurisdictional claims in published maps and institutional affiliations.

\begin{abstract}
Author details
1 Tokyo University of Technology, 1404-1 Katakuramachi, Hachioji city, Tokyo 192-0982, Japan. ${ }^{2}$ Kanagawa University, 2946 Tsuchiya, Hiratsuka city, Kanagawa 259-1293, Japan. ${ }^{3}$ Center for Advanced Intelligence Project, RIKEN, 1-4-1 Nihonbashi, Chuo-ku, Tokyo 103-0027, Japan. ${ }^{4}$ University of Shizuoka, 52-1 Yada, Suruga-ku, Shizuoka city, Shizuoka 422-8526, Japan. ${ }^{5}$ Wakayama University, 930 Sakaedani, Wakayama city, Wakayama 640-8510, Japan.
\end{abstract}

Received: 2 March 2018 Accepted: 18 June 2018

Published online: 09 July 2018

\section{References}

Aggarwal A, Deshpande A, Kannan R (2009) Adaptive sampling for k-means clustering. In: Proceedings of the 12th International Workshop and 13th International Workshop on Approximation, Randomization, and Combinatorial Optimization. Algorithms and Techniques. Springer-Verlag, Berlin, Heidelberg. pp 15-28

Berry BJL (1964) Approaches to regional analysis: A synthesis. Ann. Assoc. Am. Geogr. 54:2-11

Berry, BJL (1968) Interdependency of spatial structure and spatial behavior: A general field theory formulation. Papers Reg. Sci. Assoc. 21:2050-227

Burckhart K, Martin OJ (2012) An Interpretation of the Recent Evolution of the City of Barcelona through the Traffic Maps. J. Geogr. Inf. Syst. 4(4):298-311

Chen W, Liu W, Ke W, Wang N (2018) Understanding spatial structures and organizational patterns of city networks in China: A highway passenger flow perspective. J. Geogr. Sci. 28(4):477-494

Crucitti P, Latora V, Porta S (2006) Centrality Measures in Spatial Networks of Urban Streets. Phys. Rev. E 73(3):036,125+

Everett M, Borgatti S (1994) Regular equivalence: General theory. J. Math. Sociol. 19(1):29-52

Farmer CJQ, Fotheringham AS (2011) Network-Based Functional Regions. J. Environ. Plan. A Econ. Space 43(11):2723-2741

Fushimi T, Saito K, Ikeda T, Kazama K (2016a) Extracting and Characterizing Functional Communities in Spatial Networks. In: Proceedings of the Workshop on Artificial Intelligence for Tourism (Al4Tourism2016). pp 182-193

Fushimi T, Saito K, Ikeda T, Kazama K (2016b) Functional Cluster Extraction from Large Spatial Networks. In: ASONAM '16: Proceedings of the 2016 IEEE/ACM International Conference on Advances in Social Networks Analysis and Mining. IEEE Press, Piscataway. pp 57-62

Fushimi T, Saito K, Ikeda T, Kazama K (2017c) Fast Extraction Method of Functional Clusters from Large-Scale Spatial Networks Based on Transfer Learning. In: Proceedings of Complex Networks 2017 (The Sixth International Conference on Complex Networks and Their Applications). Springer International Publishing, Basel. pp 1210-1222 
Fushimi T, Saito K, Kazama K (2012) Extracting Communities in Networks based on Functional Properties of Nodes. In: Richards D, Kang BH (eds). Proceedings of the 12th Pacific Rim Knowledge Acquisition Workshop (PKAW2012). Springer-Verlag, Berlin, Heidelberg. pp 328-334

Gilpin S, Eliassi-Rad T, Davidson I (2013) Guided learning for role discovery (glrd): Framework, algorithms, and applications. In: Proceedings of the 19th ACM SIGKDD International Conference on Knowledge Discovery and Data Mining. ACM, New York. pp 113-121

Grigg DB (1965) The logic of regional systems. Ann. Assoc. Am. Geogr. 55:465-491

Henderson K, Gallagher B, Eliassi-Rad T, Tong H, Basu S, Akoglu L, Koutra D, Faloutsos C, Li L (2012) Rolx: Structural role extraction \& mining in large graphs. In: Proceedings of the 18th ACM SIGKDD International Conference on Knowledge Discovery and Data Mining. ACM, New York. pp 1231-1239

Henderson K, Gallagher B, Li L, Akoglu L, Eliassi-Rad T, Tong H, Faloutsos C (2011) It's who you know: Graph mining using recursive structural features. In: Proceedings of the 17th ACM SIGKDD International Conference on Knowledge Discovery and Data Mining. ACM, New York. pp 663-671

Jiang B, Duan Y, Lu F, Yang T, Zhao J (2014) Topological structure of urban street networks from the perspective of degree correlations. Environ. Plan. B Plan. Design 41(5):813-828

Jiang C, Li Y, Shao M, Jia P (2002) Accelerating clustering methods through fractal based analysis. In: KDD-2002 workshop report fractals and self-similarity in data mining: issue and approaches. ACM, New York

Leskovec J, Krause A, Guestrin C, Faloutsos C, VanBriesen J, Glance N (2007) Cost-effective outbreak detection in networks. In: Proceedings of the 13th ACM SIGKDD International Conference on Knowledge Discovery and Data Mining. ACM, New York. pp 420-429

Lin F, Cohen WW (2010) Power iteration clustering. In: Proceedings of the 27th International Conference on Machine Learning (ICML-10). International Machine Learning Society. pp 655-662

Lorrain FH, White H (1971) Structural equivalence of individuals in social networks. J. Math. Sociol. 1(1):49-80

Montis DA, Barthelemy M, Chessa A, Vespignani A (2007) The Structure of Interurban Traffic: A Weighted Network Analysis. Environ. Plan. B Plan. Des. 34(5):905-924

Newman MEJ (2004) Detecting Community Structure in Networks. Eur. Phys. J. B Condens. Matter Complex Syst. 38(2):321-330. https://doi.org/10.1140/epjb/e2004-00124-y

Opsahl T, Agneessens F, Skvoretz J (2010) Node Centrality in Weighted Networks: Generalizing Degree and Shortest Paths. Soc. Networks 32(3):245-251

Palla G, Derényi I, Farkas I, Vicsek T (2005) Uncovering the Overlapping Community Structure of Complex Networks in Nature and Society. Nature 435:814-818

Park K, Yilmaz A (2010) A Social Network Analysis Approach to Analyze Road Networks. In: Proceedings of the ASPRS Annual Conference 2010

Rossi RA, Ahmed NK (2015) Role discovery in networks. IEEE Trans Knowl Data Eng 27(4):1112-1131

Rossi RA, Gallagher B, Neville J, Henderson K (2012) Role-dynamics: Fast mining of large dynamic networks. In: Proceedings of the 21st International Conference Companion on World Wide Web. ACM, New York. pp 997-1006

Rossi RA, Gallagher B, Neville J, Henderson K (2013) Modeling dynamic behavior in large evolving graphs. In: Proceedings of the Sixth ACM International Conference on Web Search and Data Mining. ACM, New York. pp 667-676

Rosvall M, Bergstrom CT (2007) An information-theoretic framework for resolving community structure in complex networks. Proc. Natl. Acad. Sci. 104(18):7327-7331

Seidman SB (1983) Network structure and minimum degree. Soc. Networks 5(3):269-287

Vinod H (1969) Integer Programming and the Theory of Grouping. J. Am. Stat. Assoc 64(326):506-19. https://doi.org/10. 1080/01621459.1969.10500990

Wang P, Hunter T, Bayen AM, Schechtner K, Gonzalez MC (2012) Understanding Road Usage Patterns in Urban Areas. Sci. Rep. 2:1001

Yin J, Soliman A, Yin D, Wang S (2017) Depicting urban boundaries from a mobility network of spatial interactions: A case study of Great Britain with geo-located Twitter data. Int. J. Geogr. Inf. Sci. 31:1293-1313

Zhang Y, Wang X, Zeng P, Chen X (2011) Centrality Characteristics of Road Network Patterns of Traffic Analysis Zones. Transp. Res. Rec. J. Transp. Res. Board 2256:16-24

\section{Submit your manuscript to a SpringerOpen ${ }^{\circ}$ journal and benefit from:}

- Convenient online submission

- Rigorous peer review

- Open access: articles freely available online

- High visibility within the field

- Retaining the copyright to your article

Submit your next manuscript at $\gg$ springeropen.com 